\title{
The state of psychogeriatrics in different regions of the world: challenges and opportunities
}

Global population aging is pervasive, profound, and enduring, with the projected proportion of older persons reaching nearly $21 \%$ in 2050 (UNFPA and Help Age International, 2012). Accordingly, there is an increasing need for geriatric mental health services with the rapid growth of the aging population worldwide (Bragg et al., 2012).

The availability of geriatric mental health services varies substantially across the world. Some regions, or countries, such as the United Kingdom, have sophisticated geriatric mental health services and others, for example in Africa, bear the consequences of undeveloped services. Still, International Psychogeriatric Association (IPA) experts believed that an appropriate needs assessment would show both commonalities and differences in needs despite the big differences that exist in the development of geriatric mental health services in different parts of the world.

In 2011, IPA initiated the President's Forum primarily to address the contrast between the need of mental health services and the difficulties met by societies and governments in providing adequate resources to psychogeriatric professionals. In particular, each affiliate organization of IPA, which is comprised of professionals concerned primarily with activities related to those of IPA, was encouraged to share ideas how to overcome these problems and the role that IPA can play to support their organization. Second, the President's Forum also aimed to present the different possibilities and solutions to provide these services and to facilitate the cooperation by IPA between participating societies to help each other to develop new capabilities.

On September 6, 2011, when the IPA International Congress was held in the Hague, the Netherlands, representatives of 20 affiliates, mostly presidents and secretaries, attended the first President Forum and presented a report on the state of the development of geriatric psychiatry services in their countries and its perceived needs. Following the forum, all affiliates were asked to submit a written report for further analysis. Although a specific outline structure on needs, strengths, and plans for action was provided, affiliates were encouraged to use their own way to address the relevant issues. In total, 15 reports were received in document format and one in slide format; nine from the AsianPacific region, five from Europe, and 1 from South America. The prioritizing needs for the development of psychogeriatrics, strength, and available resources, and action plans are outlined below.

\section{Needs for development of psychogeriatrics}

Needs to increase the accessibility of geriatric mental health services

Nearly all affiliates reported that the accessibility of psychogeriatric mental health services is quite limited. Access to services varies by the geographic area (e.g. rural vs. urban), residence placement (e.g. home care vs. residential care), and patient characteristics (e.g. patients with intellectual disability or financially disadvantaged). Also, the type of services differs among the elderly people depending on their physical and psychological condition. For example, general practitioners (GPs) may prefer to prescribe medications rather than psychological counseling to patients with comorbidity of depression and physical conditions. Affiliates strongly agreed that there is an urgency to increase the accessibility of geriatric mental health services. Recommendations to accomplish this included promoting psychological intervention for elderly people with mental health problems, increasing liaison with general hospital services, developing outreaching services in the community, as well as supporting long-term care services. Some affiliates also agreed that community care and advocacy should be strengthened, especially through funding and networking with IPA.

For example, the Faculty of Psychiatry for Old Age, Royal Australian and New Zealand College of Psychiatrists, reported: "The position statement regarding the provision of psychological services is urgently needed. The approach of identifying, implementing, and training of optimal psychological interventions should be addressed in the position statement."

The Department of Old Age Psychiatry, Netherlands Psychiatric Association, reported: "The statement should encourage the development of effective psychological interventions available to 
elderly people for common mental health problems like anxiety and depression."

The Indonesian Psychogeriatric Association reported: "Delirium, dementia and depression are frequently diagnosed in general hospitals where elderly patients are often treated. Behavior and Psychological Symptoms of Dementia (BPSD) is the main reason to bring persons with dementia to a psychiatrist, while the diagnosis of dementia is often missed in patients with physical complaints who come to general hospital unless they receive a comprehensive assessment by the geriatric team. Delirium cases in general hospital were often undiagnosed until the medical staff experienced problems in managing patient care."

The Portuguese Gerontopsychiatric Association reported: "IPA could help to build regional and national centers for community care. In this context, Family Health Units and Community Care Units, focusing on the care centered on the citizen and their families start to be developed in Portugal. These health units will develop health care centered on the general practitioner and nurses, in connection with the Integrated Continuing Care Network (RNCCI), as well as with some Mobile Health Units."

\section{Needs to build an integrated psychogeriatric service model}

Nearly all affiliates proposed an integrated psychogeriatric service model, or integrating psychogeriatric services with other health services. It was argued that the focus of psychogeriatric service has moved to early intervention in mental illness. Care for aged people includes mental healthcare, physical healthcare, and support services. Though physical health, mental health, and "cognitive health" are intrinsically linked, they are often managed by different departments at the research, policy, or service delivery levels. More often, this leads to a lack of action regarding relevant issues. At times, the response to mental health service needs is fragmented and poorly coordinated.

In addition, outreaching services in primary care settings could also be counted as an integrative service model. The work should be strengthened, including training for medical staff in primary care settings in the near future. For example, the Faculty of Old Age Psychiatry, Royal College of Psychiatrists, United Kingdom, reported: "Although the Faculty has had official links with primary care via the Royal College of General Practitioners for many years, in reality there is little direct interaction. There is concern that despite various initiatives, dementia in the community remains under-diagnosed, depression is under-recognized and under-treated and guidance on the use of antipsychotics is not well understood. Additionally in England GPs will have a more prominent role in the commissioning of Old Age Psychiatric Services. With the introduction of the 'Health and Social Care Bill, July 2011' the way NHS is funded and managed changed totally to smaller GP led Clinical Consortia. It was suggested to examine how we can influence primary care in the clinical and commissioning field."

\section{Needs to boost the research of mental health services into old age}

Almost all affiliates agreed that research into geriatric mental health should be promoted. In some countries, although research has not yet been conducted due to limited funding, the collaboration with regional and international education centers and institutions is more promising. Meanwhile, the importance of culture, value, and perception should be considered as an indicator for psychogeriatric research and service development in a certain region. For example, the three-year QoLDEM project codirected by Prof. Edmond Chiu and Helen Chiu has stimulated the movement of improving quality of life and well-being for people with dementia and their families in Asian countries (Chiu and Chiu, 2005). For example, the Hong Kong Psychogeriatric Association (HKPGA) reported: "HKPGA organized our first Tripartite Psychogeriatric Scientific Conference with Psychogeriatric Interest Group of Chinese Society of Psychiatry (CPIG) and Taiwanese Society of Geriatric Psychiatry last year (2010) with the aim of having a sustainable collaboration among the three associations .... An agreement for the exchange program with the Japanese Society of Psychogeriatrics (JSPG) and HKPGA was signed in January 2010. The two associations each will select two delegates to present their scientific papers at their reciprocal Annual General Meetings."

As there is a lack of research supervisors specializing in psychogeriatrics and limited opportunities for research training in low-resource countries, such as China, Indonesia, and Thailand, the promotion and participation in multi-center research (both clinical and basic) led by IPA is urgently needed, either regional or international. This promotion and participation would also involve cross-cultural comparisons and epidemiology studies in different ethnicities and cultures. If possible, the IPA endorsement of research projects may provide benefits for certain affiliate organizations. These potential benefits include providing a small amount of financial support, supervising research activities, and promoting multi-center studies among affiliate countries. Additionally, IPA could recommend an expert 
panel that could consult with affiliate organizations in order to improve the quality of research and education.

Building a collaborative research network may be necessary to ensure an appropriate sample size, to make use of multidisciplinary expertise, and to have a greater impact on local service development. There are a number of current multidisciplinary, international, and collaborative research projects being conducted on longevity, healthy aging, and neuropsychiatric disorders. Also, research projects targeting different types of elderly population are needed.

For example, the Department of Old Age Psychiatry, Netherlands Psychiatric Association, reported: "Given the rise in elderly patients in need of care and the costs, future strategies are aimed to support carers optimally and develop interventions to enhance quality of life for elderly with dementia and their caregivers and to postpone nursing home admissions. Multiple research programs (Trimbos Institute, VU University, Maastricht University, Nijmegen University) in international collaboration target this topic."

CPIG reported: "People aged over 65 years old have exceeded $13 \%$ of the total population. It is critically important to maintain a healthy aging society. In addition to recent local funding to develop an intervention program on people with dementia and their caregivers, we have submitted a proposal on improving early detection and intervention of elderly people with mental problems, including subsyndromal depression, depressive disorders, mild cognitive impairment and dementia."

Incentives to conduct research may encourage more psychogeriatric mental health service providers to be involved in scientific research. For example, HKPGA reported: "The HKPGA Annual Scientific Symposium held annually in midNovember and the HKPGA Mid-year Scientific Symposium held usually in June every year. The participants would be able to learn from the local and international experts in old age psychiatry. We also invited two winners of the Young Scientist Award from the Japanese Society of Psychogeriatrics to present their research findings in the scientific symposiums.... HKPGA ran annual contest for HKPGA Research Award with the aim to encourage and reward fine research projects in Old Age Psychiatry. HKPGA picks the two winners for the exchange program with JSPG (Japanese Society of Psychogeriatrics) through the hot competition for the HKPGA Young Scientist Award."

Promoting scientific publication may be an effective way to promote scientific research. For example, the Indian Association for Geriatric Mental Health (IAGMH) reported: "[IAGMH] has a journal called Indian Journal of Geriatric Mental Health which is a peer-reviewed biannual publication with original research articles, reviews and case reports."

The Thai Society for Geriatric Psychiatry and Neuropsychiatry (TSGN) reported: "The International Psychogeriatrics (IPG) editorial team shall be encouraged to develop a positive attitude in terms of considering articles by affiliates for publication in IPG."

From a practical perspective, research ambition should be at a post-graduate level. In addition to scholarship-funded opportunities, there is a need for permanent positions for scientists to gain experience necessary to become qualified teachers. A significant part of the research needs to be connected to clinical practice. By inviting medical students, internship doctors, and psychiatry scholar candidates to collaborate in research projects, there is a greater chance for these candidates to be recruited to the research field of geriatric psychiatry.

As the challenges of psychogeriatric services are multifactorial and mostly driven by the national political agenda, the recent global financial downturn has also put great pressure on psychogeriatricspecific funding. Due to very limited financial support, the budget for psychogeriatric services has been diverted into other service departments. For example, the Faculty of Old Age Psychiatry, Royal College of Psychiatrists, United Kingdom, reported: "The community mental health teams (CMHT) consisting of Old age psychiatrists, community psychiatric nurses, nursing assistants, physiotherapists, occupational therapists, psychologists and dementia care nurses, form the backbone of mental healthcare delivery in the community. There have been significant changes in the structure and functioning of CMHTs with some places introducing crisis teams and specialized dementia/memory assessment teams. Due to the financial crisis in the last few years, these teams are facing considerable difficulty in terms of resources and some services like psychotherapy have been the victims of reduced or decommissioned care provision."

\section{Available strengths}

All present IPA affiliates represent an organized professional group in their own region, committed to supporting the development of psychogeriatric services. Most affiliates have their own connections with local service resources and to some extent have built the service capacity for mental health professionals. 
Psychogeriatric education programs have been developed by most affiliates. A few affiliates, such as the United Kingdom, Australia, and New Zealand, have adopted the accreditation system and certified the psychogeriatricians with the subspecialty training, while some are still developing the system.

For example, the Portuguese Gerontopsychiatric Association reported: "Regulation of Old Age Psychiatry education is desirable, requiring more and better care in Portugal, with the development of geriatric centers of excellence, in order to allow an official certification emphasized by the importance of developing the competency (according to WPA Consensus/WHO Geriatric Psychiatry), and with accreditation by international organizations, like IPA and EAGP."

The Indonesian Psychogeriatric Association reported: "Psychogeriatric education module in the medical doctor curriculum has been developed since 2005 in FMUI and other education centers including nursing, psychology, and social work. A psychiatry registrar specialty includes a 2-month internship in Psychogeriatrics in FMUI. They should achieve competency in managing common elderly mental health problems in the society and hospital settings."

The Taiwanese Society of Geriatric Psychiatry (TSGP) reported: "Although the certificate of subspecialty is just honorary, our members enrolled in TSGP basically have high motivation for involvement in the activities and are enthusiastic about advanced learning. In addition, as our members are all board-certificated psychiatrists, the quality of clinical competence and scientific skills is high. With systematic guidance, adequate support, and more incentive measures, the development of old age psychiatry will probably flourish."

Also, it has been recognized that IPA taskforces are playing a greater role in promoting psychogeriatric services and that this work should be continued. For example, TSGN reported: "The BPSD taskforce has achieved its goal in terms of introducing a new BPSD package and a capacity taskforce is planning to develop its first set of guidelines."

\section{Proposed plan of action}

\section{Promoting links with primary care}

Geriatric mental health services should provide care integrated with primary care or community healthcare. This would be less expensive and more accessible. As outlined in the needs of psychogeriatric service, the integrated service model may help to promote the well-being of the elderly people living in the community.
The Faculty of Old Age Psychiatry, Royal College of Psychiatrists, United Kingdom, reported: "The main points to be considered are - linking with primary care, supporting GPs in effective clinical service for elderly people, supporting the faculty members, discussing with GPs about the cost involved in Diagnosis (Payment by Results); what would be the cost if mental health related disorders are not diagnosed early, the advantages of improved links with primary care, the disadvantages of these links, the barriers in achieving the links and to form an action plan."

The Portuguese Gerontopsychiatric Association reported: "In spite of the recent reform in Mental Health Care in Portugal, shifting the orientation of mental health policy from traditional psychiatric hospitals toward community primary care, psychiatric services in hospitals or centers, there is a lack of effective answers, namely coordination between the social and public health sectors."

The Indonesian Psychogeriatric Association reported: "In the primary care level, the elderly are served by Puskesmas (primary healthcare centers) which are spreading in every sub-district across the country through the Santun Lansia program. In practice, it is expected that puskesmas have a good collaboration with hospital and other specialized clinic because the mental health experts are still scarce. In the near future, training for medical staff in primary care is set as a priority to empowering and increasing knowledge in psychogeriatrics including the skill of counseling and caring for persons with dementia."

CPIG reported: "CPIG will conduct the second national survey on mental health service resources. The data will be mapped for strategic planning of mental health service. It was also proposed that IPA could provide expertise in promoting and developing the community-based mental health service model in China."

\section{Achieving a global recognition of geriatric psychiatry as a subspecialty}

The level of qualification in geriatric psychiatry needs to be improved both in primary and specialized healthcare. As the pivot for promoting psychogeriatrics through the propagation of educational programs, activities, training courses, and the certificate examinations, IPA affiliates may be able to empower their members with updated knowledge and skills, raise the standards of practice, and keep all members competent in geriatric psychiatry. Therefore, IPA could provide support to promote recognition of the subspecialty of Geriatric Psychiatry in regions where it has not been adequately valued. 
For example, the Taiwanese Society of Geriatric Psychiatry reported: "Although a serious examination system has been built up, the department of health in the government has still paid little attention to this subspecialty and does not admit its merit. Certificate of specialist for old age psychiatry is actually honorary, and there is absolutely no physical benefit from this expertise. The situation might weaken the motivation of junior psychiatrists to become psychogeriatricians and hinder their future development. With systematic guidance, adequate support, and more incentive measures, the development of old age psychiatry will probably flourish."

The Portuguese Gerontopsychiatric Association reported: "A lot of work needs to be done, namely with structured education programmes as well as the recognition of a sub-specialization of Internal Medicine or General Medicine or even a definition of a competency, namely in gerontopsychiatry. ... support in recognizing competency in Geriatric Psychiatry, namely accreditation of this subspecialty by training psychiatrists in prestigious institutions and support of scientific societies, like IPA."

The Spanish Psychogeriatric Association (SEPG) reported: "Psychiatric subspecialties are not recognized yet in Spain. ... The new training curriculum of the specialty of Psychiatry for Resident doctors envisages a development of 4 specific areas of training: Psychiatry for Children and Adolescents, Gerontopsychiatry, Psychiatry of Alcoholism and other Addictions, and Psychotherapy. ... The aim is to achieve the recognition of the subspecialty of Geriatric Psychiatry. A prerequisite is to get the point 2 , training psychiatrists in this field and accrediting such training by prestigious institutions, such as international scientific societies, e.g., IPA."

In addition, IPA could organize a working group to study the current core curriculum of existing subspecialty training programs and seek a consensus on the required and optional criteria for subspecialty certificate. Additional work needs to be done, namely with structured education programs as well as the recognition of a sub-specialization of internal medicine or general medicine or even a definition of a competency, namely in gerontopsychiatry. Once the recognition of subspecialty of geriatric psychiatry is achieved, we could move to the next step, i.e., recruiting and training psychiatrists in this field and accrediting such training by prestigious institutions, such as international scientific societies.

\section{Building a stronger leading role for IPA}

It is generally known that IPA provides opportunities for its affiliates and members to meet and learn from each other, as well as exchange recommendations and advice on research projects. IPA also sup- ports the development of different taskforces. However, there is not a strong relationship between IPA and its affiliates. As a result, IPA should collect and manage detailed data regarding these taskforces, such as how many there are, their plans, their achievements and what they want from other nontaskforce members, so that greater levels of participation can be achieved. IPA should also consider contacting other affiliates, so that they too can participate in the different taskforces.

It is highly recommended that IPA could take several actions to promote psychogeriatric services, including building international collaborative research network, providing expertise and consultation for local training and research projects to ensure the quality of the activities. IPA could encourage and partially support its prestigious members to visit underrepresented countries for their scientific conferences, continuous medical educational program, or even subspecialty training courses. Some affiliates would like IPA to promote research among affiliate organizations through the endorsement of research projects by giving financial support, supervising research activities, and promoting multi-centered studies among affiliate countries.

To reduce planning silos, it would be a practical action to develop a framework useful for policymakers and service developers highlighting the relationship between geriatric mental health service and relevant social service.

\section{Conclusion}

This is the first time that IPA has made the effort to explore the state of psychogeriatrics in different regions of the world. It is intended to be an annual exercise that will reflect the strengths and weaknesses in the field of geriatric mental health, as well as identify challenges that confront it in different regions of the world in this ever-changing healthcare environment. There are a number of interesting issues presented in the affiliates' reports in the Presidents' Forum.

We identified needs to develop psychogeriatrics from 20 different regions of the world. The main common trends will be to increase accessibility, develop an integrated service model, and boost research capacity.

The differences are found in the capacity and resources in the different regions. Some countries have a greater level of training experience in the subspecialty of geriatric psychiatry, while other countries have very limited or even no subspecialty training. The capacity varies across the disciplines. 
Physicians have more training than nurses, social workers, psychologists, and other providers.

Resources also differ in different areas of the world. At first glance, it may seem that geriatric mental health service resources vary with the economic situation. That is not always the case. Some countries that are very rich have very limited geriatric resources, while others that are resource-limited have already placed geriatric mental health on the agenda of their health service. Therefore, some of the differences may not only be dependent on the financial resources and availability but also on political will.

We believe these findings commit IPA to develop an action plan based on the priorities discussed. The recognition of geriatric psychiatry as a subspecialty is on the rise in Europe (Toot et al., 2012). To promote this worldwide, the support of IPA is critically needed. Follow-up reports will allow us to evaluate the ability of IPA to take action and the global impact that these actions may have in the field of geriatric mental health service.

There are several limitations in the report. Information from North America, where psychogeriatrics has been well developed compared with other regions in the world, was not included in the analysis (Lieff et al., 2003; Andrew and Shea, 2010; Bragg et al., 2012). Additionally, we have made every effort to obtain a report from Japan, one of the most aged countries in the world. In Japan, geriatric psychiatry is now a rapidly growing subspecialty (Maeda, 2009). Unfortunately, our attempts were not successful. Therefore, the trends in psychogeriatrics and current aging situations and challenges could not be accurately represented.

\section{Participating affiliates}

Royal Australian and New Zealand College of Psychiatrists - Faculty of Psychiatry for Old Age (RANZCP-FPOA) (Roderick McKay), Psychogeriatric Interest Group, Chinese Society for Psychiatry (CPIG) (Xin Yu and Huali Wang), Hong Kong Psychogeriatric Association (Siu-Wa Li and Helen Chiu), Indian Association for Geriatric Mental Health (IAGMH) (Charles Pinto), Indonesian Psychogeriatric Association (Martina WS Nasrun), Korean Association of Geriatric Psychiatry (KAGP) (Maeng Je Cho and Changsu Han), Taiwanese Society of Geriatric Psychiatry (YungJen Yang and Te-Jen Lai), Thai Society for Geriatric Psychiatry and Neuropsychiatry (TSGN) (Nahathai Wongpakaran and Pichet Udomratn), The Dementia Association (Thailand) (Vorapun Senanarong), Portuguese Gerontopsychiatric Association (Lia Fernandes), The Spanish Psychogeriatric Association (Raimundo Mateos), Faculty of Old
Age Psychiatry, Royal College of Psychiatrists, UK (Anand Ramakrishnam), Netherlands Psychiatric Association, Department of Old Age Psychiatry (Max L. Stek), Norwegian Psychiatric Association (Knut Engedal), European Association of Geriatric Psychiatry (EAGP) (Ralf Ihl), and Brazilian Association of Geriatric Neuropsychiatry (Sónia Brucki).

\section{Conflict of interest}

None.

HuAli WANG, ${ }^{1}$ LIA FERNANDES, ${ }^{2}$ SUSAN OSTER, ${ }^{3}$ MASATOSHI TAKEDA, ${ }^{4}$ HENRY BRODATY $^{5}$ AND JACOBO E. MINTZER ${ }^{6}$, on behalf of the President Forum Working Group of International Psychogeriatric Association

${ }^{1}$ Key Laboratory for Mental Health, Ministry of Health, Peking University Institute of Mental Health, Beijing 100191, China

${ }^{2}$ Faculty of Medicine, Psychiatry Department of CHSJ

Porto, University of Porto, Porto, Portugal

${ }^{3}$ International Psychogeriatric Association, Chicago, Illinois, USA

${ }^{4}$ Department of Psychiatry, Osaka University Graduate

School of Medicine, Osaka, Japan

${ }^{5}$ Centre for Healthy Brain Ageing, University of New

South Wales, Sydney, Australia

${ }^{6}$ Department of Health Studies, College of Health

Professions, Medical University of South Carolina,

Charleston, South Carolina, USA

Email: huali_wang@bjmu.edu.cn

\section{Acknowledgment}

The authors thank Professor Mary-Jo Good for advice on qualitative analysis.

\section{References}

Andrew, M. H. and Shea, C. (2010). Geriatric psychiatry education in Canada: the pathway to subspecialty recognition. International Psychogeriatrics, 22, 919-926.

Bragg, E. J., Warshaw, G. A., Cheong, J., Meganathan, K. and Brewer, D. E. (2012). National survey of geriatric psychiatry fellowship programs: comparing findings in 2006/07 and 2001/02 from the American Geriatrics Society and Association of Directors of Geriatric Academic Programs' Geriatrics Workforce Policy Studies Center. The American fournal of Geriatric Psychiatry, 20, 169-178.

Chiu, H. and Chiu, E. (2005). Dementia care in Asia. International Psychogeriatrics, 17, 1-2.

Lieff, S. J., Warshaw, G. A., Bragg, E. J., Shaull, R. W., Lindsell, C. J. and Goldenhar, L. M. (2003). Geriatric psychiatry fellowship programs in the United States: findings from the Association of Directors of Geriatric 
Academic Programs' longitudinal study of training and practice. The American fournal of Geriatric Psychiatry, 11, 291-299.

Maeda, K. (2009). "Geriatric psychiatry" as a rapidly growing subspecialty [in Japanese]. Seishin Shinkeigaku Zasshi, 111, 938-944.
Toot, S., Orrell, M., Rymaszewska, J. and Ihl, R. (2012). A survey of geriatric psychiatry training across Europe. International Psychogeriatrics, 24, 803-808.

UNFPA and Help Age International. (2012). Ageing in the Twenty-First Century: A Celebration and A Challenge. New York: UNFPA and Help Age International. 